\title{
Response to "Neck pain and proprioception deficit influence the cervical motion assessed by instantaneous axis of rotation"
}

\author{
Chung Kee Chung ${ }^{1}$ (D) Chi Heon Kim ${ }^{1}$ \\ Received: 6 April 2018 / Accepted: 11 April 2018 / Published online: 21 April 2018 \\ (C) Springer-Verlag GmbH Austria, part of Springer Nature 2018
}

Wang et al. commented on the effect of proprioception recovery and postoperative neck pain on the change of instantaneous axis of rotation (IAR).

I totally agree that proprioception plays an important role in controlling IAR and that 6 months was not enough for the recovery of proprioception. The main objective of our study was to show the change of IAR after relief of neck and arm pain. If the cervical anatomical structure were severely damaged, restoration of a normal IAR may not occur even with the improvement of pain. Our study showed that the normal range of IARs was maintained with minimally invasive motion preservation surgeries (posterior percutaneous endoscopic foraminotomy, PECF) 6 months after surgery. As Wang et al. mentioned, preservation of normal anatomical structure is important for stable cervical mobility. Therefore, PECF is a good alternative in this regard. Because our study was a retrospective one, proprioceptive function was not assessed. When taking recovery time into consideration, the result may change with a longer follow-up period. Evaluation of proprioception in a prospective manner is necessary to provide the ultimate outcome of motion preservation surgeries.
Regarding residual neck pain, neck pain was higher after posterior open foraminotomy (PCF) than PECF or total disc replacement (TDR), although statistical significance was not reached. I also suppose that postoperative neck pain has an influence on the abnormal neck motion. However, the difference of neck pain was less than 1 out of 10 numeric pain score and the range was within mild pain. The usual mean clinically important difference (MCID) of neck pain is 2.5 [1]. Hence, the influence of residual pain with a minor difference may or may not be reflected in IAR.

Finally, as Wang et al. pointed out, we need a larger and longer follow-up study to elucidate the effect of proprioception and postoperative mild neck pain on the range of motion after motion preservation surgeries on the neck.

\section{Reference}

1. Pool JJ, Ostelo RW, Hoving JL, Bouter LM, de Vet HC (2007) Minimal clinically important change of the Neck Disability Index and the Numerical Rating Scale for patients with neck pain. Spine (Phila Pa 1976) 32:3047-3051
Chung Kee Chung

chungc@snu.ac.kr

1 College of Medicine, Seoul National University, Seoul, South Korea 